\title{
ESR/ERS white paper on lung cancer screening
}

\author{
Hans-Ulrich Kauczor ${ }^{1,9} \cdot$ Lorenzo Bonomo $^{2} \cdot$ Mina Gaga $^{3} \cdot$ Kristiaan Nackaerts $^{4}$. \\ Nir Peled ${ }^{5}$ Mathias Prokop ${ }^{6}$ - Martine Remy-Jardin ${ }^{7}$ • Oyunbileg von Stackelberg ${ }^{1,9}$. \\ Jean-Paul Sculier ${ }^{8}$ on behalf of the European Society of Radiology (ESR) and the \\ European Respiratory Society (ERS)
}

Received: 27 February 2015 / Accepted: 27 February 2015 / Published online: 1 May 2015

(C) The Author(s) 2015. This article is published with open access at Springerlink.com

\begin{abstract}
Lung cancer is the most frequently fatal cancer, with poor survival once the disease is advanced. Annual low-dose computed tomography has shown a survival benefit in screening individuals at high risk for lung cancer. Based on the available evidence, the European Society of Radiology and the European Respiratory Society recommend lung cancer screening in comprehensive, quality-assured, longitudinal programmes within a clinical trial or in routine clinical practice at certified multidisciplinary medical centres. Minimum requirements include: standardised operating procedures for low-dose image acquisition, computer-assisted nodule evaluation, and positive screening results and their management; inclusion/exclusion criteria; expectation management; and smoking cessation programmes. Further refinements are recommended to increase quality, outcome and costeffectiveness of lung cancer screening: inclusion of risk
\end{abstract}

This is an official white paper of the European Society of Radiology (ESR) and the European Respiratory Society (ERS), published in European Radiology [DOI: 10.1007/s00330-015-3697-0] and the European Respiratory Journal [DOI: 10.1183/09031936.00033015]. It was approved by the ESR Executive Council in February 2015, and by the ERS Steering Committee on 12 March 2015.

Hans-Ulrich Kauczor

hans-ulrich.kauczor@med.uni-heidelberg.de

1 Dept of Diagnostic and Interventional Radiology, University Hospital Heidelberg, Heidelberg, Germany

2 Institute of Radiology, A. Gemelli University Hospital, Rome, Italy

3 7th Resp. Med. Dept and Asthma Center, Athens Chest Hospital, Athens, Greece

4 KU Leuven-University of Leuven, University Hospitals Leuven, Department of Respiratory Diseases/Respiratory Oncology Unit, Leuven, Belgium models, reduction of effective radiation dose, computerassisted volumetric measurements and assessment of comorbidities (chronic obstructive pulmonary disease and vascular calcification). All these requirements should be adjusted to the regional infrastructure and healthcare system, in order to exactly define eligibility using a risk model, nodule management and a quality assurance plan. The establishment of a central registry, including a biobank and an image bank, and preferably on a European level, is strongly encouraged.

Key points

- Lung cancer screening using low dose computed tomography reduces mortality.

- Leading US medical societies recommend large scale screening for high-risk individuals.

- There are no lung cancer screening recommendations or reimbursed screening programmes in Europe as of yet.

5 Davidoff Cancer Center, Rabin Medical Center, Tel Aviv University, Tel Aviv, Israel

6 Department of Radiology and Nuclear Medicine, Radboud University Medical Center, Nijmegen, Netherlands

7 Department of Thoracic Imaging, Hospital Calmette (EA 2694); CHRU et Universite de Lille, Lille, France

8 Thoracic oncology, Institut Jules Bordet, Université Libre de Bruxelles, Brussels, Belgium

9 Translational Lung Research Center, Member of the German Lung Research Center, Heidelberg, Germany 
- The European Society of Radiology and the European Respiratory Society recommend lung cancer screening within a clinical trial or in routine clinical practice at certified multidisciplinary medical centres.

- High risk, eligible individuals should be enrolled in comprehensive, quality-controlled longitudinal programmes.

Keywords Lung cancer screening - Low-dose CT . Pulmonary nodule $\cdot$ Overdiagnosis

$\begin{array}{ll}\text { Abbreviations and acronyms } \\ \text { AATS } & \text { American Association for Thoracic Surgery } \\ \text { ACCP } & \text { American College of Chest Physicians } \\ \text { ACS } & \text { American Cancer Society } \\ \text { ALA } & \text { American Lung Association } \\ \text { ASCO } & \text { American Society of Clinical Oncology } \\ \text { CTDI } & \text { Vol } \\ \text { COPD } & \text { Chrome CT dose index } \\ \text { CXR } & \text { Chest X-Ray } \\ \text { DANTE } & \text { Detection and Screening of Early Lung Cancer } \\ & \text { by Novel Imaging Technology and Molecular } \\ & \text { Essays } \\ \text { DLST } & \text { Danish Lung Screening Trial } \\ \text { IASLC } & \text { International Association for the Study of Lung } \\ & \text { Cancer } \\ \text { ITALUNG } & \text { Italian lung study } \\ \text { LDCT } & \text { Low-dose computed tomography } \\ \text { LLP } & \text { Liverpool Lung Project } \\ \text { LUSI } & \text { German Lung Screening and Intervention trial } \\ \text { mAs } & \text { Milliampere-seconds } \\ \text { MILD } & \text { Multicentric Italian Lung Detection } \\ \text { mSv } & \text { Millisievert } \\ \text { mGy } & \text { Milligray } \\ \text { NCCN } & \text { National Comprehensive Cancer Network } \\ \text { NELSON } & \text { NEderlands-Leuvens Screening ONderzoek } \\ \text { NLST } & \text { National Lung Screening Trial } \\ \text { PLCO } & \text { Prostate Lung, Colorectal, and Ovarian } \\ & \text { randomazed trial } \\ \text { SSAC } & \text { Strategic Screening Advisory Committee } \\ \text { UKLS } & \text { UK Lung Screen } \\ \text { USPSTF } & \text { US Preventive Services Task Force } \\ \text { VDT } & \text { Volume doubling time } \\ & \end{array}$

\section{Introduction}

Lung cancer causes 1.37 million deaths per year worldwide, which represents $18 \%$ of all cancer deaths [1]. Within the European Union, lung cancer is the most frequently fatal cancer, leading to over 266,000 deaths yearly and accounting for $20.8 \%$ of all cancer deaths [2]. Definitive surgery in the early stages is the most effective treatment for lung cancer. However, most patients are diagnosed at an advanced, and thus non-curable, disease stage. Survival time decreases significantly with progression of disease, with a 5-year survival time declining from $50 \%$ for clinical stage IA to $43 \%, 36 \%$, $25 \%, 19 \%, 7 \%$ and $2 \%$ for stages IB, IIA, IIB, IIIA, IIIB and IV, respectively [3]. Moreover, Shi et al. [4] reported a 5-year survival rate of more than $80 \%$ in 185 surgically treated patients with peripheral small-sized lung cancers $(2 \mathrm{~cm}$ or less) after lobectomy and lymph node dissection. In particular, the 5-year survival rate increased with smaller tumour size: 80 $\%$ in tumours $1.6-2.0 \mathrm{~cm}$ in diameter, $85 \%$ in tumours 1.0 $1.5 \mathrm{~cm}$ in diameter and $100 \%$ in tumours $<1.0 \mathrm{~cm}$ in diameter, respectively. It is therefore crucial to detect lung cancer early, before symptoms occur and while curable therapy is still achievable.

During the past decade, several studies focused on the yield of low dose computed tomography (LDCT)-based screening for lung cancer. In total, roughly 100,000 high-risk individuals were screened for lung cancer by LDCT. The largest randomised trial, the US-based National Lung Screening Trial (NLST), has shown a survival benefit for annual LDCT, with a $20 \%$ reduction of the lung cancer related mortality, whereas the all-cause mortality decreased by $6 \%$. However, there is still some debate associated with the appropriate algorithm with which to select the screening cohort, as well as with how exactly the images should be read. The second largest study, the European NEderlands-Leuvens Screening ONderzoek (NELSON) trial, will be finalised by the end of 2015 and will add insight, probably with a more accurate screening algorithm and lower rate of false positivity, as discussed by Shlomi et al. [5].

As the results of this large European study are pending and the screening algorithms used in published studies have not been universal, there are numerous issues that should be taken into consideration before starting an LDCT screening programme in Europe. This paper will review the current status of lung cancer screening, and provide recommendations for the standards and additional evidence required.

\section{Status quo}

\section{Results of the current trials}

The NLST is the first randomised, controlled lung cancer screening trial in current and former smokers (> 30 packyears) aged between 55 and 74 years to show a significant reduction in lung-cancer-specific mortality [6]. The computed tomography (CT) screening arm of the trial involved 26,722 participants who received three yearly screening rounds of LDCT. The control arm involved 26,732 participants who received three yearly screening rounds using chest 
radiographs. After a follow-up period of approximately 6.5 years, participants in the CT screening arm were $20 \%$ less likely to die from lung cancer than those in the control arm. A $6 \%$ reduction in overall mortality was also observed within the 6.5-year period. In the CT screening arm, 356 participants died from lung cancer, whereas the number in the corresponding radiography arm was 443 [6]. In an additional evaluation 1 year later, these numbers had increased to 469 in the CT arm and 552 in the radiography arm, which corresponds to a $15 \%$ reduction [7]. These results suggest that LDCT finds more cancers, most of them being in stage IA ( $>50 \%)$ and approximately $10 \%$ in stage IB [8]. Still, $43 \%$ (469 out of 1089) of those patients who developed lung cancer died of lung cancer. The overall screening effort meant that 320 participants had to be screened to prevent one lung cancer death within the 6.5year follow-up period [6].

The Dutch-Belgian NELSON trial is the largest European randomised controlled trial with at-risk participants based on age and smoking history randomly selected from population registries. The first outcome data are expected in 2016. The trial involves 7577 participants in the CT screening arm and compares them to 7871 participants in the control arm [9]. Apart from a smoking cessation programme, no intervention was offered in the control arm. Published results are available from smaller randomised controlled trials from Denmark (DLST) and Italy (Italung, DANTE and MILD). These trials involved approximately 1000-2000 patients in each arm [10]. Published results suggest no advantage for lung cancer screening. In fact, DLST and MILD even found a trend towards higher mortality in the yearly CT screening arms [11, 12]. Other current randomised controlled trials are the German Lung Screening and Intervention (LUSI) trial and the UK Lung Screening (UKLS) trial [13, 14].

\section{Current recommendations}

There is a wide range of acceptance of the general lung cancer screening algorithm using LDCT across the globe; however, different degrees of modification from the NLST algorithm seem to be required (Table 1) [5].

From February 2012, the Lung Cancer Screening Panel of the National Comprehensive Cancer Network (NCCN) in the USA recommended annual LDCT screening of all high-risk individuals between the age of 55 and 74 years, as defined in the NLST [15]. However, the NCCN guidelines expanded the NLST criteria based on non-randomised studies and observational data. Individuals 50 years of age or older with a tobacco smoking history of 20 or more pack-years and one additional risk factor should be screened annually. The suggested additional risk factors were history of cancer, history of lung disease [chronic obstructive pulmonary disease (COPD) or pulmonary fibrosis], family history of lung cancer, radon exposure and occupational exposure. The NCCN currently does not advise screening of individuals at moderate and low risk for lung cancer, or for individuals with exposure to secondhand smoke [16].

A collaborative initiative of the American Cancer Society [17], the American College of Chest Physicians (ACCP), the American Society of Clinical Oncology [18], and the NCCN published a review of LDCT screening for lung cancer, together with clinical practice guidelines, in May 2012 [10]. They adopt the NLST eligibility criteria, but note that the duration and frequency of screening remain undetermined [18]. In June 2012, guidelines for lung cancer screening were issued by the American Association for Thoracic Surgery (AATS) [19], expanding the criteria beyond the NLST. The AATS guidelines consider the amount of tobacco exposure and age to be the most important risk factors, and therefore do not restrict screening to patients who quit smoking in the previous 15 years. Since the risk of lung cancer does not decrease after 3 years of screening, the AATS recommends annual LDCT screening for high-risk patients from age 55 to 79 years. They consider that level 2 evidence is enough to advise screening for smokers 50-79 years of age with a 20 pack-year smoking history or other factors that produce a cumulative $\geq 5$ $\%$ risk of developing lung cancer over the following 5 years. Based on AATS consensus opinion (level 3 evidence), patients treated for primary bronchogenic carcinoma who have completed 4 years of radiographic surveillance without evidence for recurrence should also be screened. In January 2013, the American Cancer Society published guidelines that recommend annual lung screening by LDCT based on the NLST eligibility criteria until the age of 74 years [20]. In May 2013, the ACCP published its third edition guidelines of diagnosis and management of lung cancer, including a recommendation concerning lung cancer screening [21, 22]. Annual screening with LDCT for individuals who meet the NLST eligibility criteria is recommended (grade 2B; weak recommendation, moderate level of evidence).

In December 2013, the United States Preventive Services Task Force (USPSTF) developed their recommendation statement [23], which was published in March 2014 [24], supporting LDCT lung cancer screening for healthy adults between 55 and 80 years of age with a smoking history of 30 pack-years or more and who have smoked within the previous 15 years. The number of years needed for screening is not specified, but screening should be discontinued once a person has not smoked for 15 years or develops a health problem that substantially limits the life expectancy or the ability or willingness to have curative lung surgery (grade B recommendation). Under the Affordable Care Act [25], any procedure that receives a grade $\mathrm{B}$ recommendation from the US Preventive Services Task Force (USPSTF) has to be covered by private insurers without co-payment. Most insurers in the USA follow the recommendations of the task force, and pay for those services. In April 2014, the US federal agency 


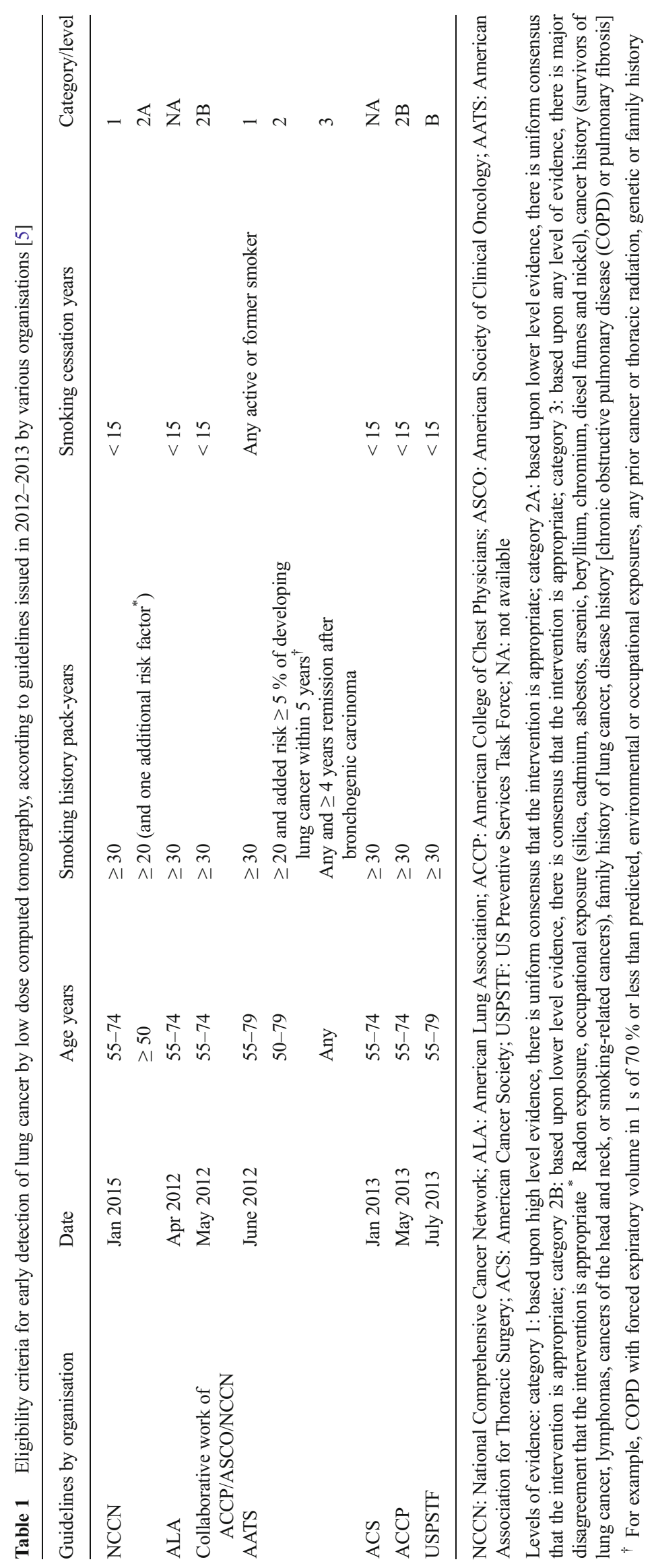


Center for Medicare and Medicaid services (CMS) advisory panel voted against covering lung cancer screening [26]. Key concerns were the high false-positive rate of CT screening, indication creep outside of the intended screening population, inability to assure quality scans with low radiation dose, and consistent interpretation and diagnostic work-up in routine practice. In February 2015, in contrast to the recommendations of the agency's advisory board, Medicare announced its decision to start covering annual lung cancer screening once per year for long-time smokers at high risk for the disease [27]. CMS experts require that screening candidates are between ages 55 and 77 years, have no signs or symptoms of lung disease, have tobacco smoking history of at least 30 pack-years and are current smokers or ex-smokers who have quit smoking within the previous 15 years. For the initial screen, the beneficiary must receive a written order for LDCT lung cancer screening obtained during a "lung cancer screening counselling and shared decision-making visit" from a physician, physician assistant, nurse practitioner or clinical nurse specialist. CMS also gives details for that visit, radiologist eligibility criteria and imaging centre eligibility criteria [27]. In Europe, there are no lung cancer screening recommendations or reimbursed screening programmes so far.

\section{Challenges}

\section{Pre-test probability}

Tobacco smoking is a major risk factor for lung cancer, as shown by many epidemiological studies. Other less important risk factors are passive (second-hand) smoking, occupational exposure, environmental exposure, residential radon exposure, presence of COPD and family history of lung, head and neck cancer. A meta-analysis of ten case-control studies including 7609 cases and 10,431 controls shows an increase of the relative risk of lung cancer in the European population with active smoking (versus ex-smokers), with duration and amount of smoking and the cumulative dose of pack-years [28]. The recommendations for the NELSON trial are based on data from the Cancer Prevention Study II (CPS II) [29]. According to this data, a smoking history of 30 years or more in individuals older than 55 years and a consumption of at least one pack of cigarettes a day correspond to a lung cancer incidence of at least 300 per 100,000. The rate of lung cancer diagnosed for those selected populations in recent randomised trials by LDCT are summarised in Table 2 .

More data from other cohorts are described in the systematic review published by Bach et al. [10]. The rate of lung cancer diagnosed ranges between 0.8 and $2.2 \%$ initially and between $2.4 \%$ and $4.7 \%$ in 34-78 months of follow-up. Those figures can be taken as the pre-test probability.

\section{Overdiagnosis}

The detection of small lesions confirmed to be malignant, but which do not grow, spread, or cause death is referred to as overdiagnosis. This includes patients who are destined to die from another cause, e.g., comorbidity or an unexpected event, in addition to slow growing/non-spreading cancers [10]. Overdiagnosis represents an important potential harm of screening, since it incurs additional cost, anxiety and morbidity associated with the cancer treatment. During earlier screening trials using chest radiographs in the Mayo and the Czechoslovakian randomised trials, substantially more cancers $(20 \%)$ were detected in the screened than in the unscreened group [30, 31]. Nearly all of the excess cancers detected in the screened group in the Mayo clinical trial were early stage cancers. However, the failure to detect early stage cancers in the control group was without apparent ill effect: the control group experienced no excess number of lung cancer deaths [30]. The results were generally confirmed by the Czechoslovakian study. Both studies suggest that screening is detecting "excess" lesions, which probably would not progress to advanced/lethal disease [30, 31]. The PLCO trial [17] examined 155,000 subjects in the general population and found 18 excess lung cancers in the chest radiography group (compared with no chest radiography group) after 6 years of follow-up ( 2 years after screening ended) and 76 lung cancers after 13 years of follow-up. Data from the same trial, evaluating overdiagnosis among a high-risk population only, showed a cumulative incidence of lung cancer of 606 per 100,000 person-years in the chest radiography group and 608 per 100,000 person-years in the usual care group after 6 years of follow-up.

The overdiagnosis rate for LDCT screening cannot yet be estimated [24]. The NLST data shows a persistent gap of about 120 excess lung cancers in the LDCT versus the chest radiography arm, but further follow-up is needed [32]. In both groups, the percentage of stage IA and stage IB lung cancers was high. Relative to the issue of overdiagnosis, fewer stage IV cancers were detected in the LDCT group than in the chest radiography group at the second and third screening rounds in the DANTE trial, where 2472 subjects were screened with chest radiography and sputum cytology at baseline and randomised afterwards to yearly LDCT or clinical follow-up. Lung cancer prevalence in the control chest radiography arm was $0.67 \%(\mathrm{n}=8)$ and $50 \%$ of these patients had stage I cancer, while the prevalence in the CT group was $2.19 \%(\mathrm{n}=$ 28 ) with $57 \%$ stage I cancer, respectively. It has to be noted that 13 of the 28 LDCT lung cancer cases already had abnormal chest radiography findings at baseline [33].

Still, while most lung cancer prevention experts think lung cancer screening leads to overdiagnosis, many clinicians believe it does not [34]. Death rates from lung cancer imply that essentially all histological foci of lung cancer pose a threat to 
Table 2 Selection criteria, number of enrolled individuals and the rate of diagnosed lung cancer of major randomised controlled trials

\begin{tabular}{|c|c|c|c|c|}
\hline \multirow[t]{2}{*}{ Study } & \multicolumn{2}{|c|}{ Selection criteria } & \multirow[t]{2}{*}{ Patients screened n (follow-up) } & \multirow{2}{*}{$\begin{array}{l}\text { Lung cancer diagnosed at initia } \\
\text { screening (total in follow- up) }\end{array}$} \\
\hline & Age years & Tobacco smoking (delay since weaning) & & \\
\hline DLCST & $50-70$ & $\geq 20$ pack-years $(0-9$ years $)$ & 2052 (58 months) & $0.8 \%(3.4 \%)$ \\
\hline DANTE & $\begin{array}{l}60-74 \\
\text { Only men }\end{array}$ & $\geq 20$ pack-years $(0-9$ years $)$ & 1276 (34 months) & $2.2 \%(4.7 \%)$ \\
\hline ITALUNG & $55-69$ & $\geq 20$ pack-years (active or former) & 1406 (36 months) & $1.5 \%(2.8 \%)$ \\
\hline MILD & $\geq 49$ & $\geq 20$ pack-years $(0-9$ years $)$ & $\begin{array}{l}1190^{*} \text { (120 months) } \\
1186^{\dagger} \text { (53 months) }\end{array}$ & $0.8 \%(2.4 \%)$ \\
\hline NELSON & $50-75$ & $\geq 15$ pack-years ${ }^{\ddagger}(0-9$ years $)$ & 7907 (60 months) & $0.9 \%(2.6 \%)$ \\
\hline NLST & $55-74$ & $\geq 30$ pack-years $(0-15$ years $)$ & 26722 (78 months) & $1.1 \%(2.4 \%)$ \\
\hline
\end{tabular}

${ }^{*}$ Annual computed tomography; ${ }^{\dagger}$ Biannual computed tomography; ${ }^{\star}$ NELSON inclusion criteria: number of cigarettes smoked is $\geq 15$ per day for 25 years $\mathrm{OR} \geq 10$ cigarettes per day for 30 years AND still smoking or having quit $<10$ years ago.

health, irrespective of their CT phenotype or how they are discovered. In the NLST, the size of the nodule and whether it is solid or sub-solid mattered. However, whether this appearance is linked to higher overdiagnosis probability remains to be concluded. Based on the Pan-Canadian early Detection of Lung Cancer Study (PanCan), McWilliams et al. [35] presented a model to predict a cancerous pulmonary nodule (versus benign). Predictors for cancer were older age, female sex, family history of lung cancer, emphysema and larger nodule size, location of the nodule in the upper lobe, part-solid nodule type, lower nodule count and spiculation. Adopting such a model may direct the clinicians in their follow-up management.

\section{Risk models}

Risk models help to increase pre-test probability and reduce overdiagnosis. They improve the patient selection in order to define populations with higher pre-test probabilities: the Liverpool Lung Project (LLP) risk prediction model is used in the UKLS screening trial; the PLCO2012 (Prostate, Lung, Colorectal, and Ovarian) randomised trial and the NLST trial. The former two studies predict lung cancer detection, while the latter predicts death by lung cancer (Table 3).

Recently, de Koning et al. [39] published a study estimating the harms and benefits of lung cancer screening for efficient lung cancer screening policies. They used five separately developed micro-simulation models calibrated to the two largest randomised, controlled trials on lung cancer screening [17, 39]. Those models were independently developed at five institutions: Erasmus Medical Center (Rotterdam), Fred Hutchinson Cancer Research Center (Seattle), Massachusetts General Hospital (Boston), Stanford University (Stanford), and University of Michigan (Ann Arbor). All account for the individual's age-specific, smoking-related risk for lung cancer, date and stage of lung cancer diagnosis, the corresponding lung cancer mortality and the individual's life expectancy in the presence and absence of screening. The most advantageous strategy identified is the annual screening from ages 55 through 80 years for ever-smokers with a smoking history of at least 30 pack-years and ex-smokers with less than 15 years since quitting. That approach would lead to $50 \%$ of cases of cancer being detected at an early stage (stage I/II), 575 screening examinations per lung cancer death averted, a $14 \%$ reduction in lung cancer mortality, 497 lung cancer deaths averted, and 5250 life-years gained per the 100,000member cohort. Harms would include 67,550 false-positive test results, 910 biopsies or surgeries for benign lesions, and 190 overdiagnosed cases of cancer (3.7\% of all cases of lung cancer).

Thus far, there are no good risk predictors for nonsmokers and no convincing data to recommend screening. Lung cancer in never smokers is the seventh leading cause of cancer mortality, and therefore is a significant cause of death worldwide. The main risk factors include age, environmental tobacco exposure, cooking fumes, inherited genetic susceptibility, occupational and environmental exposure to carcinogens, hormonal factors, pre-existing lung disease and oncogenic viruses [40]. Nonsmall cell lung cancer (NSCLC) in never smokers is clinically characterised by an increased incidence in females and a higher occurrence of adenocarcinoma in comparison to NSCLC in ever smokers in both surgical patients and nonresectable advanced stage patients [41]. Even though those factors are known, there is no beneficial screening programme for lung cancer among this population.

\section{False positives and complications during work-up}

With modern multidetector CT, pulmonary nodules are detectable at a size of less than $2 \mathrm{~mm}$. Small nodules are extremely common, but the vast majority of these nodules are benign. Given this fact, the definition of a positive screening result determines the number of false-positive results. On average, about $25 \%$ of the thoracic surgical procedures performed 
Table 3 Risk prediction models used in different lung cancer screening trials

\begin{tabular}{|c|c|c|c|}
\hline Model & Risk factors included & $\begin{array}{l}\text { Period of prediction of lung } \\
\text { cancer diagnosis or death }\end{array}$ & Reference for algorithm \\
\hline LLP (detection) & $\begin{array}{l}\text { Age } \\
\text { Sex } \\
\text { Years of smoking } \\
\text { Family history of lung cancer by age of affected relatives } \\
\text { History of a previous cancer } \\
\text { History of pneumonia } \\
\text { History of exposure to asbestos }\end{array}$ & 5 years & Raji et al. [36] \\
\hline PLCO (detection) & $\begin{array}{l}\text { Age } \\
\text { Race/ethnicity } \\
\text { Education } \\
\text { Body mass index } \\
\text { Chronic obstructive pulmonary disease } \\
\text { Personal history of cancer } \\
\text { Family history of lung cancer } \\
\text { Smoking status (current versus former) } \\
\text { Smoking intensity (average cigarettes/day) } \\
\text { Smoking duration } \\
\text { Smoking quit time }\end{array}$ & 6 years & Tammemägi et al. [37] \\
\hline NLST (death) & $\begin{array}{l}\text { Age } \\
\text { Sex } \\
\text { Ethnicity } \\
\text { Body-mass index } \\
\text { Pack-years of smoking } \\
\text { Years since smoking cessation } \\
\text { Presence of emphysema } \\
\text { First-degree relative with lung cancer }\end{array}$ & 5 years & Kovalchik et al. [38] \\
\hline
\end{tabular}

during the various randomised controlled lung cancer screening trials were done for benign nodules [21]. If there are fewer false-positive nodules, there is less need for further workup and lower risk of complications, especially from invasive diagnostic examinations including surgery.

The definition of a positive screening result differed substantially between the NLST and most European trials. The NLST defined any non-calcified nodule with a maximum diameter $\geq 4 \mathrm{~mm}$ as a positive screening result [6]. As a consequence, the number of false-positive scans was high: $27 \%$ of scans in the first two screening rounds, of which $96 \%$ were false-positive. According to the NLST nodule management algorithm, these suspicious nodules needed further work-up: either a follow-up LDCT for nodules of 4$10 \mathrm{~mm}$, or a referral to a pulmonologist for nodules > $10 \mathrm{~mm}$ in maximum diameter [6].

The NELSON and some other European trials used a threshold of approximately $10 \mathrm{~mm}$ diameter $\left(50 \mathrm{~mm}^{3}\right.$ volume) for a positive screening result, but also established an indeterminate group of nodules measuring $5-10 \mathrm{~mm}$ in diameter $\left(50-500 \mathrm{~mm}^{3}\right.$ volume) that required earlier follow-up than the yearly screening interval [42]. Only if significant growth ( $>25 \%$ volume change) was found, were these nodules considered a positive screening result. By using this approach, the number of scans with positive screening results was reduced from $27 \%$ in the NLST to $2.7 \%$ in the NELSON, and the false-positives could be reduced substantially from $>95 \%$ in the NLST to approximately $50 \%$ in the NELSON $[8,43]$.

Recently, new criteria for the follow-up of pulmonary nodules, such as LungRADS and LU-RADS, have been presented in order to increase the positive predictive value in CT screening with minimum effect on sensitivity for the detection of malignancy $[44,45]$.

The size of a nodule was measured in most screening trials, like the NLST, as the largest diameter of a pulmonary nodule [6]. This approach suffers from a substantial inter-reader and intra-reader variability, which can be reduced by applying volumetric techniques, as used in the NELSON and other more recent trials. Non-actionable nodules were defined as those with benign morphology (e.g., calcification), small size $\left(<50 \mathrm{~mm}^{3}\right)$, and lack of or very slow growth of the solid 
component of a nodule with a volume doubling time (VDT) > 600 days. Indeterminate nodules were defined as nodules with a volume of the solid component between 50 and $500 \mathrm{~mm}^{3}$, sub-solid nodules with a diameter of the ground glass component $>10 \mathrm{~mm}$, or solid nodules with a VDT between 400 and 600 days. Actionable nodules were defined as solid components $>500 \mathrm{~mm}^{3}$, more than $20 \%$ growth in diameter of a ground glass component, or VDT $<400$ days of a solid component [42]. Non-actionable, reportable nodules were kept on regular (yearly) follow-up, indeterminate nodules were put on a more rapid follow-up of 3-6 months, while actionable nodules led to direct medical workup.

Increasing knowledge about the CT phenotypes of screendetected pulmonary nodules with different biologic behaviours will lead to a better estimation of their probability of malignancy, and help to decrease the amount of additional follow-up scans and workup examinations [46], e.g., perifissural nodules were demonstrated to have a high likelihood of being benign [47, 48].

For the invasive diagnostic work-up of small nodules, the value of white light fibrebronchoscopy is very limited [49], but newer diagnostic endoscopic techniques, such as endobronchial ultrasound-guided biopsy with mini probe or electromagnetic navigation bronchoscopy, might be more promising. For some peripheral nodules $(>1 \mathrm{~cm})$, transthoracic CT-guided biopsy or primary resection by video-assisted thoracoscopic surgery for diagnostic and therapeutic reasons may be recommended [50]. The risk of serious complications (pneumothorax requiring drainage, cardiorespiratory complications during anaesthesia, infection or haemorrhage) not only relates to the invasiveness of the diagnostic procedure itself, but also to the patient's functional status [51]. Subjects eligible for LDCT screening will present themselves mostly with a high comorbidity risk, due to COPD or chronic cardiovascular disease [46, 52].

Adhering to a certified high quality radiology plan for LDCT screening will minimise radiation exposure for screening participants. Further, the adherence to a pulmonary nodule management plan based on nodule diameter, volume and growth rate will help to increase safety for lung cancer screening participants, mostly by decreasing the total amount of diagnostic investigations they will need to undergo in order to determine the nature of their screen-detected lung nodules. Moreover, a lower amount of false-positive lesions with a decreased number of additional diagnostic investigations may finally help to decrease participant's anxiety and psychological stress during lung cancer screening [53].

\section{Radiation exposure}

The vast majority of lung cancer screening trials were designed more than a decade ago. The LDCT protocols were simply achieved by reducing the fixed tube load of diagnostic
CT from typically $100-300 \mathrm{mAs}$ to $10-40 \mathrm{mAs}$. A CT dose index $\left(\mathrm{CTDI}_{\mathrm{vol}}\right)$ of $2-3 \mathrm{mGy}$ was used as a target for NLST $[54,55]$. Similar values were used in the NELSON and the various other European trials. The resulting effective dose is roughly $40 \%$ of these values for males and $50 \%$ for females, resulting in $1-1.3 \mathrm{mSv}$ for a $\mathrm{CTDI}_{\mathrm{vol}}$ of $2.5 \mathrm{mGy}$. The organ dose $(\mathrm{mSv})$ to the lung or to the breast can be roughly estimated using $1.5 \times \mathrm{CTDI}_{\mathrm{vol}}$. Precise numbers vary depending on scanner type, and in particular on the pre-filtering of the Xray spectrum.

With recent improvements in detector technology, automated exposure control techniques and iterative image reconstruction, a further substantial decrease in radiation exposure of 80 $\%$ to a level around $0.2 \mathrm{mSv}$ is possible without impairing image quality [56]. However, radiation exposure will always have to be higher in obese individuals than in normal weight individuals because of the difference in X-ray absorption. Excessive reduction of radiation dose will lead to image quality degradation with either high image noise or loss of image details, which will especially affect sub-solid lesions. These are the limiting factors for further dose reduction.

Radiation risk in the age range of 40 to 60 years is mainly determined by the organ dose to the lungs. Apart from the breast in premenopausal women, other organs have a much lower contribution to excess cancer risk [57]. Radiation exposure and smoking appear to have an additive effect on cancer risk [58]. This means that the excess risk for developing radiation-induced lung cancer may be twice as high in smokers as in never-smokers [59].

Given an effective dose of $1.3 \mathrm{mSv}$ for women and 1.0 $\mathrm{mSv}$ for men, the excess lifetime cancer risk was estimated to be $0.02 \%$ in male smokers and $0.05 \%$ in female smokers if three yearly screening rounds were performed [60]. Risks did not change whether the starting age for screening was 30, 40 or 50 years. This implies that radiation risk becomes important only if the pre-test risk for lung cancer is small. Given a baseline cancer risk of $0.8-2.2 \%$ in the various screening trials, the risk-benefit ratio is very favourable. Even if the number of screening examinations increases from three to 24 , the excess lifetime cancer risk induced by radiation remains below the baseline cancer risk, but it increases with age [38].

Radiation risk grows strongly if follow-up scans are performed using standard clinical protocols (old equipment 4-18 $\mathrm{mSv}$, new equipment 2-4 mSv [61]) instead of screening with LDCT settings (new equipment $0.2 \mathrm{mSv}$ [56]). For this reason, the work-ups of screen-detected nodules should remain within the screening programme as long as possible [62].

\section{Cost effectiveness}

The cost-effectiveness of the screening intervention is one of the major considerations for those who are responsible for screening guidelines, practice measures and insurance 
coverage [63]. Varying results on the cost-effectiveness of lung cancer screening have been reported [64-67]. In their recent publication, the NLST reports reasonable costeffectiveness of LDCT screening of lung cancer [68]. LDCT screening as performed in the NLST trial costs $\$ 81,000$ per quality-adjusted life year (QALY) gained (95\% CI \$52,000$186,000)$. Screening trials that cost less than $\$ 100,000$ per QALY are considered cost-effective. Incremental cost effectiveness ratio (ICER) is the ratio of the change in costs to incremental benefits of a therapeutic intervention or treatment [69]. The NLST ICER was $\$ 52,000$ (95\% CI $\$ 34,000-106$, 000). However, the ICER results of the NLST were highly sensitive to base-case assumptions. For example, if the reduction in mortality from causes other than lung cancer was included in the calculation, the QALY fell to $\$ 54,000$. QALY increased to more than $\$ 100,000$ when the cost of future care was increased. Moreover, estimated cost-effectiveness varied in the subgroup analysis. Screening with LDCT was much more cost-effective in women than in men and among the groups with a higher risk of lung cancer. Whether screening performed in different countries in Europe will be costeffective depends on exactly how the screening will become implemented [68], and on which respective cost structures and reimbursement policies will be used.

\section{Expectation management}

Expectation management is crucial for a successful CT screening programme. It is important for three main reasons: 1) giving participants the ability to understand the benefits and potential harms, 2) reducing anxiety in case a nodule is found and 3) reducing litigation and its chances for success. Screening is very likely to reduce a participant's risk of dying from lung cancer. However, a substantial group of participants will still die from lung cancer. Most cancers found will be in a treatable stage (60-80\% stage I) - but not all $[8,70]$. Some cancers may grow so slowly that they will not be life-limiting and treating them may be unnecessary (overdiagnosis) [39]. Screening is known to miss nodules present on LDCT [71]. The annual screening programme will pick up nodules missed on earlier scans, which reduces the risk of missed nodules developing into untreatable cancer. As small nodules are extremely common, it is very likely that a nodule will be found. LDCT is not optimally suited for the detection and diagnosis of many other chest diseases. However, incidental findings leading to unnecessary workup, costs and complications may occur.

Information given to participants, clinicians not involved in screening and the public should be clearly understandable. Informed consent is important because of the dangers of undetected cancers, overdiagnosis or complications due to workup or treatment of screen-detected lesions. The participants should be aware of the incidental finding policy of the screening programme.

\section{Broaden the scope}

CT-based screening can provide a more global approach of a smoker's lung and associated comorbidities, which are associated with poor health status and prognosis $[72,73]$. Regarding smoker's lung features, namely airway disease and emphysema, they can be easily depicted and categorised according to the proposed CT phenotypes [74]. In addition, it is also possible to detect interstitial lung abnormalities in cigarette smokers, as recently observed in $8 \%$ of the COPDGene cohort (194 out of 2416) [75]. While such abnormalities are visually assessed, recent approaches favour automated extraction and quantification of morphological changes in order to refine COPD phenotyping [76] and help predict clinical impairment [77].

Smokers have a highly increased risk for the development of cardiovascular diseases, which also coexist with COPD [72]. Since lung cancer screening examinations use noncontrast and non-ECG-gated acquisitions, precise analysis of mural changes at the level of coronary arteries, as well as thoracic aorta, remains beyond the scope of such examinations. However, several studies have documented the feasibility of an imaging approach combining lung cancer and quantification of coronary artery calcium in a single chest CT study $[78,79]$. Quantification of coronary and aortic calcium volumes in lung cancer screening CT images has recently been shown to help predict cardiovascular risk. Such an approach might prove useful in the reduction of cardiovascular morbidity and mortality, and may enhance the cost-effectiveness of CT-based screening in heavy smokers [80]. Other key targets such as calcifications at the level of heart valves and/or supraaortic arteries could also be included. Osteoporosis is also increasingly recognised as a major comorbidity that can be picked up on LDCT of the chest.

The specificity of a screening programme might also be increased by including non-imaging, non-invasive biomarkers to allow a better discrimination between benign versus malignant conditions. Examination of serum and plasma biomarkers shows some evidence supporting the rationale of using these biomarkers for risk stratification of screendetected lung nodules [81-83]. However, there are only few biomarkers that could be implemented immediately. We encourage the community to further investigate in this area and define it as an urgent unmet need in the field of lung cancer.

\section{Suggestions}

The European Society of Radiology and the European Respiratory Society are recommending lung cancer screening 
in comprehensive, quality-assured programmes within a clinical trial or in routine clinical practice at certified multidisciplinary medical centres. Based on the results and experience of completed and on-going lung cancer screening activities, we suggest the following minimum requirements for the implementation of lung cancer screening:

- Accredited medical centres with multidisciplinary expertise and access to trained professionals, including, as a minimum, radiologists, pulmonologists, oncologists, pathologists and chest surgeons.

- Strong smoking cessation programme and experienced staff providing effective cessation and long-term abstinence advice.

- Longitudinal comprehensive screening programme throughout the age interval of eligibility, covering the complete protocol, including work-up, follow-up and potential re-entry, also offering an appropriate expectation management. Single-round screening is discouraged.

- Inclusion criteria: age between 55 and 80 years, tobacco smoking history of at least 30 pack-years, and current smoker or ex-smoker who has quit smoking within the last 15 years.

- Exclusion criteria: comorbidities precluding curative therapy and lack of consent to undergo curative therapy.

- Standardised operating procedures for image acquisition, nodule evaluation, positive screening results and their management, monitoring of false-positive results and rate of iatrogenic complications, and appropriate follow-up.

- Computer-assisted nodule evaluation and documentation. Identical measurement software is required for the followup. Volumetric measurements are preferred over diameter measurements.

- Multidetector LDCT with at least 16 detector rows providing isotropic high spatial resolution (slice thickness of about $1 \mathrm{~mm}$ with an increment of 0.7 $\mathrm{mm}$ ) and an effective dose between $1 \mathrm{mSv}$ for normal sized individuals and not more than $3 \mathrm{mSv}$ for obese individuals.

- Collection and submission of lung cancer screening data to a lung cancer screening registry. The set-up of a European lung cancer screening registry including biobank and image bank is encouraged.

We also recommend the implementation of the following measures in order to increase quality, outcome and costeffectiveness of lung cancer screening:

- To increase the pre-test probability by using a risk model and considering additional risk factors.

- To reduce the effective radiation dose to less than $1 \mathrm{mSv}$ per CT examination for all participants.
- To use volumetric measurements for the assessment of growth rate (tumour doubling time) in order to reduce the rate of false-positives.

- To use computer-assisted systems for automated detection, optimised measurements and follow-up, providing structured reports on nodule volume, localisation, phenotype and standard operating procedure-based suggestions for further management plan.

- To adapt screening intervals based on refined risk models.

- To include additional CT findings such as COPD and vascular calcification.

- To include and study biomarkers to better define screening subgroups and refine nodule management.

\section{Conclusion}

Lung cancer is a devastating disease with poor survival once the disease is advanced. As the main risk factor for lung cancer is smoking, there is an urgent need to advocate against smoking and encourage cessation. There are accumulated data supporting the survival benefit for screening of individuals at high risk for early detection of lung cancer using LDCT. Based on the available evidence, we have summarised the key elements necessary for a comprehensive lung cancer screening programme in Europe, including minimum requirements and recommended refinements. These should be adjusted to the national infrastructure and healthcare system in order to exactly define eligibility using a risk model, nodule management and quality assurance plan. The establishment of a central registry, including a biobank and an image bank, preferably on a European level, is strongly encouraged.

Acknowledgments We acknowledge the support from the ESR office, in particular Chiara Manavella.

Conflict of interest: Disclosures can be found alongside the online version of this article at erj.ersjournals.com

Open Access This article is distributed under the terms of the Creative Commons Attribution Noncommercial License which permits any noncommercial use, distribution, and reproduction in any medium, provided the original author(s) and the source are credited.

\section{References}

1. World Health Organization. Cancer. Fact Sheet Number 297. www. who.int/mediacentre/factsheets/fs297/en/ Last updated February 2015. Date last accessed: February 10, 2015.

2. Eurostat Press Office. 1 in 4 Deaths Caused by Cancer in the EU28. 2014; http://ec.europa.eu/eurostat/documents/2995521/6131615/325112014-BP-EN/aab2c2d3-aed9-430a-a561-e188b8ef49d8 Date last updated: November 25, 2014. Date last accessed: February 10, 2015.

3. Goldstraw P, Crowley J, Chansky K et al (2007) The IASLC Lung Cancer Staging Project: proposals for the revision of the TNM stage 
groupings in the forthcoming (seventh) edition of the TNM Classification of malignant tumours. J Thorac Oncol 2:706-714

4. Shi CL, Zhang XY, Han BH et al (2011) A clinicopathological study of resected non-small cell lung cancers $2 \mathrm{~cm}$ or less in diameter: a prognostic assessment. Med Oncol 28:1441-1446

5. Shlomi D, Ben-Avi R, Balmor GR et al (2014) Screening for lung cancer: time for large-scale screening by chest computed tomography. Eur Respir J 44:217-238

6. Aberle DR, Adams AM, Berg CD et al (2011) Reduced lung-cancer mortality with low-dose computed tomographic screening. N Engl J Med 365:395-409

7. Pinsky PF, Church TR, Izmirlian G et al (2013) The National Lung Screening Trial: results stratified by demographics, smoking history, and lung cancer histology. Cancer 119:3976-3983

8. Aberle DR, DeMello S, Berg CD et al (2013) Results of the two incidence screenings in the National Lung Screening Trial. N Engl J Med 369:920-931

9. van Iersel CA, de Koning HJ, Draisma G et al (2007) Risk-based selection from the general population in a screening trial: selection criteria, recruitment and power for the Dutch-Belgian randomised lung cancer multi-slice CT screening trial (NELSON). Int J Cancer 120:868-874

10. Bach PB, Mirkin JN, Oliver TK et al (2012) Benefits and harms of CT screening for lung cancer: a systematic review. JAMA 307: 2418-2429

11. Saghir Z, Dirksen A, Ashraf H et al (2012) CT screening for lung cancer brings forward early disease. The randomised Danish Lung Cancer Screening Trial: status after five annual screening rounds with low-dose CT. Thorax 67:296-301

12. Pastorino U, Rossi M, Rosato V et al (2012) Annual or biennial CT screening versus observation in heavy smokers: 5 -year results of the MILD trial. Eur J Cancer Prev 21:308-315

13. Baldwin DR, Duffy SW, Wald NJ et al (2011) UK Lung Screen (UKLS) nodule management protocol: modelling of a single screen randomised controlled trial of low-dose CT screening for lung cancer. Thorax 66:308-313

14. Becker N, Motsch E, Gross ML et al (2012) Randomized study on early detection of lung cancer with MSCT in Germany: study design and results of the first screening round. J Cancer Res Clin Oncol 138:1475-1486

15. Wood DE, Eapen GA, Ettinger DS et al (2012) Lung cancer screening. J Natl Compr Canc Netw 10:240-265

16. Wood DE, Kazerooni E, Baum SL et al (2015) Lung cancer screening, version 1. J Natl Compr Canc Netw 13:23-34

17. Oken MM, Hocking WG, Kvale PA et al (2011) Screening by chest radiograph and lung cancer mortality: the Prostate, Lung, Colorectal, and Ovarian (PLCO) randomized trial. JAMA 306: 1865-1873

18. Bach PB, Mirkin JN, Oliver TK, et al. The Role of CT Screening for Lung Cancer in Clinical Practice. The Evidence Based Practice Guideline of the American College of Chest Physicians and the American Society for Clinical Oncology. Alexandria, ACSO, 2012; Available from www.asco.org/institute-quality/role-ctscreening-lung-cancer-clinical-practice-evidence-based-practiceguideline

19. Jaklitsch MT, Jacobson FL, Austin JH et al (2012) The American Association for Thoracic Surgery guidelines for lung cancer screening using low-dose computed tomography scans for lung cancer survivors and other high-risk groups. J Thorac Cardiovasc Surg 144:33-38

20. Wender R, Fontham ET, Barrera E Jr et al (2013) American Cancer Society lung cancer screening guidelines. CA Cancer J Clin 63: 107-117

21. Detterbeck FC, Mazzone PJ, Naidich DP et al (2013) Screening for lung cancer: diagnosis and management of lung cancer, 3rd ed:
American College of Chest Physicians evidence-based clinical practice guidelines. Chest 143:e78S-e92S

22. Detterbeck FC, Lewis SZ, Diekemper R et al (2013) Executive summary: diagnosis and management of lung cancer, 3rd ed: American College of Chest Physicians evidence-based clinical practice guidelines. Chest 143:7S-37S

23. US Preventive Services Task Force. Screening for Lung Cancer. Rockville, USPSTF Program Office, 2013. Available from www. uspreventiveservicestaskforce.org/uspstf13/lungcan/lungcanfact. pdf

24. Moyer VA (2014) Screening for lung cancer: U.S. Preventive Services Task Force recommendation statement. Ann Intern Med 160:330-338

25. US Department of Health and Human Services. Key Features of the Affordable Care Act. www.hhs.gov/healthcare/rights/ Date last updated: November 18, 2014. Date last accessed: February 10, 2015.

26. Centers for Medicare and Medicaid Services. Lung Cancer Screening with Low Dose Computed Tomography. www.cms. gov/medicare-coverage-database/details/medcac-meeting-details. aspx?MEDCACId=68 Date last accessed: February 10, 2015.

27. Centers for Medicare and Medicaid Services. Decision Memo for Screening for Lung Cancer with Low Dose Computed Tomography (LDCT) (CAG-00439 N). Baltimore, Centers for Medicare and Medicaid Services, 2015. Available from www.cms.gov/ medicare-coverage-database/details/nca-decision-memo.aspx? NCAId $=274 \& b c=$ AAAAAAAAAgAAAA $\% 3 d \% 3 d \&$

28. Simonato L, Agudo A, Ahrens W et al (2001) Lung cancer and cigarette smoking in Europe: an update of risk estimates and an assessment of inter-country heterogeneity. Int J Cancer 91:876-887

29. Department US (1990) of Health and Human Services. From the Centers for Disease Control. Health benefits of smoking cessation. JAMA 264:1930

30. Marcus PM, Bergstralh EJ, Fagerstrom RM et al (2000) Lung cancer mortality in the Mayo Lung Project: impact of extended followup. J Natl Cancer Inst 92:1308-1316

31. Kubik AK, Parkin DM, Zatloukal P (2000) Czech Study on Lung Cancer Screening: post-trial follow-up of lung cancer deaths up to year 15 since enrollment. Cancer 89:2363-2368

32. Church TR, Black WC, Aberle DR et al (2013) Results of initial low-dose computed tomographic screening for lung cancer. N Engl J Med 368:1980-1991

33. Infante M, Cavuto S, Lutman FR et al (2009) A randomized study of lung cancer screening with spiral computed tomography: threeyear results from the DANTE trial. Am J Respir Crit Care Med 180: 445-453

34. Bach PB (2008) Overdiagnosis in lung cancer: different perspectives, definitions, implications. Thorax 63:298-300

35. McWilliams A, Tammemagi MC, Mayo JR et al (2013) Probability of cancer in pulmonary nodules detected on first screening CT. N Engl J Med 369:910-919

36. Raji OY, Duffy SW, Agbaje OF et al (2012) Predictive accuracy of the Liverpool Lung Project risk model for stratifying patients for computed tomography screening for lung cancer: a case-control and cohort validation study. Ann Intern Med 157:242-250

37. Tammemagi MC, Katki HA, Hocking WG et al (2013) Selection criteria for lung-cancer screening. N Engl J Med 368:728-736

38. Kovalchik SA, Tammemagi M, Berg CD et al (2013) Targeting of low-dose CT screening according to the risk of lung-cancer death. $\mathrm{N}$ Engl J Med 369:245-254

39. de Koning HJ, Meza R, Plevritis SK et al (2014) Benefits and harms of computed tomography lung cancer screening strategies: a comparative modeling study for the U.S. Preventive Services Task Force. Ann Intern Med 160:311-320 
40. McCarthy WJ, Meza R, Jeon J et al (2012) Chapter 6: lung cancer in never smokers: epidemiology and risk prediction models. Risk Anal 32(suppl 1):S69-S84

41. Yano T, Haro A, Shikada Y et al (2011) Non-small cell lung cancer in never smokers as a representative 'non-smoking-associated lung cancer': epidemiology and clinical features. Int J Clin Oncol 16: 287-293

42. Xu DM, Gietema H, de Koning H et al (2006) Nodule management protocol of the NELSON randomised lung cancer screening trial. Lung Cancer 54:177-184

43. van Klaveren RJ, Oudkerk M, Prokop M et al (2009) Management of lung nodules detected by volume CT scanning. N Engl J Med 361:2221-2229

44. McKee BJ, Regis SM, McKee AB et al (2015) Performance of ACR Lung-RADS in a Clinical CT Lung Screening Program. J Am Coll Radiol 12:273-276

45. Manos D, Seely JM, Taylor J et al (2014) The Lung Reporting and Data System (LU-RADS): a proposal for computed tomography screening. Can Assoc Radiol J 65:121-134

46. Field JK, Hansell DM, Duffy SW et al (2013) CT screening for lung cancer: countdown to implementation. Lancet Oncol 14:e591-e600

47. Xu DM, van der Zaag-Loonen HJ, Oudkerk M et al (2009) Smooth or attached solid indeterminate nodules detected at baseline CT screening in the NELSON study: cancer risk during 1 year of follow-up. Radiology 250:264-272

48. Ahn MI, Gleeson TG, Chan IH et al (2010) Perifissural nodules seen at CT screening for lung cancer. Radiology 254:949-956

49. van't Westeinde SC, Horeweg N, Vernhout RM et al (2012) The role of conventional bronchoscopy in the workup of suspicious CT scan screen-detected pulmonary nodules. Chest 142:377-384

50. Prokop M (2014) Lung cancer screening: the radiologist's perspective. Semin Respir Crit Care Med 35:91-98

51. Van't Westeinde SC, Horeweg N, De Leyn P et al (2012) Complications following lung surgery in the Dutch-Belgian randomized lung cancer screening trial. Eur J Cardiothorac Surg 42: 420-429

52. Marshall HM, Bowman RV, Yang IA et al (2013) Screening for lung cancer with low-dose computed tomography: a review of current status. J Thorac Dis 5(suppl 5):S524-S539

53. Mulshine JL, D'Amico TA (2014) Issues with implementing a high-quality lung cancer screening program. CA Cancer J Clin 64:352-363

54. Aberle DR, Berg CD, Black WC et al (2011) The National Lung Screening Trial: overview and study design. Radiology 258:243253

55. Cagnon CH, Cody DD, McNitt-Gray MF et al (2006) Description and implementation of a quality control program in an imagingbased clinical trial. Acad Radiol 13:1431-1441

56. Katsura M, Matsuda I, Akahane M et al (2013) Model-based iterative reconstruction technique for ultralow-dose chest CT: comparison of pulmonary nodule detectability with the adaptive statistical iterative reconstruction technique. Invest Radiol 48:206-212

57. National Research Council. Health Risks from Exposure to Low Levels of Ionizing Radiation: BEIR VII. Washington, The National Academies Press, 2006.

58. Pierce DA, Sharp GB, Mabuchi K (2003) Joint effects of radiation and smoking on lung cancer risk among atomic bomb survivors. Radiat Res 159:511-520

59. Furukawa K, Preston DL, Lonn S et al (2010) Radiation and smoking effects on lung cancer incidence among atomic bomb survivors. Radiat Res 174:72-82

60. Berrington de Gonzalez A, Kim KP, Berg CD (2008) Low-dose lung computed tomography screening before age 55: estimates of the mortality reduction required to outweigh the radiation-induced cancer risk. J Med Screen 15:153-158
61. Mettler FA Jr, Huda W, Yoshizumi TT et al (2008) Effective doses in radiology and diagnostic nuclear medicine: a catalog. Radiology 248:254-263

62. McCunney RJ, Li J (2014) Radiation risks in lung cancer screening programs: a comparison with nuclear industry workers and atomic bomb survivors. Chest 145:618-624

63. Sox HC (2011) Better evidence about screening for lung cancer. $\mathrm{N}$ Engl J Med 365:455-457

64. Marshall D, Simpson KN, Earle CC et al (2001) Potential costeffectiveness of one-time screening for lung cancer (LC) in a high risk cohort. Lung Cancer 32:227-236

65. Wisnivesky JP, Mushlin AI, Sicherman N et al (2003) The costeffectiveness of low-dose CT screening for lung cancer: preliminary results of baseline screening. Chest 124:614-621

66. Mahadevia PJ, Fleisher LA, Frick KD et al (2003) Lung cancer screening with helical computed tomography in older adult smokers: a decision and cost-effectiveness analysis. JAMA 289: 313-322

67. Manser R, Dalton A, Carter R et al (2005) Cost-effectiveness analysis of screening for lung cancer with low dose spiral CT (computed tomography) in the Australian setting. Lung Cancer 48:171-185

68. Black WC, Gareen IF, Soneji SS et al (2014) Cost-effectiveness of CT screening in the National Lung Screening Trial. N Engl J Med 371:1793-1802

69. Folland SGA, Stano M (2010) Economic Effiencey and CostBenefit Analysis. The Economics of Health and Health Care. Prentice Hall, Boston

70. Horeweg N, van der Aalst CM, Thunnissen E et al (2013) Characteristics of lung cancers detected by computer tomography screening in the randomized NELSON trial. Am J Respir Crit Care Med 187:848-854

71. Devaraj A (2015) Missed cancers in lung cancer screening-more than meets the eye. Eur Radiol 25:89-91

72. Vestbo J, Hurd SS, Agusti AG et al (2013) Global strategy for the diagnosis, management, and prevention of chronic obstructive pulmonary disease: GOLD executive summary. Am J Respir Crit Care Med 187:347-365

73. Masala S, Magrini A, Taglieri A et al (2014) Chronic obstructive pulmonary disease (COPD) patients with osteoporotic vertebral compression fractures (OVCFs): improvement of pulmonary function after percutaneous vertebroplasty (VTP). Eur Radiol 24:15771585

74. Hansell DM, Bankier AA, MacMahon H et al (2008) Fleischner Society: glossary of terms for thoracic imaging. Radiology 246: 697-722

75. Washko GR, Lynch DA, Matsuoka S et al (2010) Identification of early interstitial lung disease in smokers from the COPDGene Study. Acad Radiol 17:48-53

76. Castaldi PJ (2013) San Jose Estepar R, et al. Distinct quantitative computed tomography emphysema patterns are associated with physiology and function in smokers. Am J Respir Crit Care Med 188:1083-1090

77. Estepar RS, Kinney GL, Black-Shinn JL et al (2013) Computed tomographic measures of pulmonary vascular morphology in smokers and their clinical implications. Am J Respir Crit Care Med 188:231-239

78. Shemesh J, Henschke CI, Shaham D et al (2010) Ordinal scoring of coronary artery calcifications on low-dose CT scans of the chest is predictive of death from cardiovascular disease. Radiology 257: 541-548

79. Budoff MJ, Nasir K, Kinney GL et al (2011) Coronary artery and thoracic calcium on noncontrast thoracic CT scans: comparison of ungated and gated examinations in patients from the COPD Gene cohort. J Cardiovasc Comput Tomogr 5:113-118 
80. Mets OM, Vliegenthart R, Gondrie MJ et al (2013) Lung cancer screening CT-based prediction of cardiovascular events. JACC Cardiovasc Imaging 6:899-907

81. Pecot CV, Li M, Zhang XJ et al (2012) Added value of a serum proteomic signature in the diagnostic evaluation of lung nodules. Cancer Epidemiol Biomarkers Prev 21:786-792
82. Daly S, Rinewalt D, Fhied C et al (2013) Development and validation of a plasma biomarker panel for discerning clinical significance of indeterminate pulmonary nodules. J Thorac Oncol 8:31-36

83. Jett JR, Peek LJ, Fredericks L et al (2014) Audit of the autoantibody test, EarlyCDT(R)-lung, in 1600 patients: an evaluation of its performance in routine clinical practice. Lung Cancer 83:51-55 